\title{
Needs assessment to identify content for simulation- based curricula in emergency medicine
}

To the editor:

We read with interest the study by Kester-Greene et al. that identified content of a simulation-based curriculum in adult emergency medicine. $^{1}$

Performing a needs assessment to identify what procedures to develop is an important first step when developing a complete curriculum. ${ }^{2}$ It is timely that training programs are not planned based on educators' personal preferences or coincidences with the risk of purchasing expensive training equipment that ends up gathering dust because of a lack of demand.

The authors performed a classical Delphi method, in which topics were rated for importance, resulting in a list divided into "core" and "extended" curricular topics. Prioritization of key procedures is an important step, but the methodology used does not provide knowledge on why these were prioritized. It was stated that the panel could provide reasons for their choices. Generally, comment boxes in questionnaires are used sparingly, and the current study does not report any of these qualitative data. The panelists might rate a procedure well suited for simulation because it is rarely performed, making it difficult to obtain and maintain competence in the clinical setting or because it is a frequent procedure that every physician in adult emergency medicine must master. Similarly, they might exclude topics that they believe are difficult to teach because of cost, a lack of well-suited equipment, or a lack of local expertise. These non-reported reasons for prioritization reduce the transparency of the traditional Delphi approach. In the current study, some topics were surprisingly excluded, and the authors had to speculate on possible explanations.

We have performed both national and international needs assessment in 13 specialties using a modified Delphi method with the aim to not only identify procedures for simulation-based training but also, more importantly, prioritize them based on transparent criteria. ${ }^{3}$ In Delphi round two, we used a needs assessment formula (NAF) to explore factors that could determine the importance of developing a simulation-based training program for a given procedure. ${ }^{4}$ These include four factors:

1. Frequency of procedure

2. Number of physicians

3. Impact on patients

4. Feasibility

The NAF scores produce a preprioritized list that the panel would then explore in round three, giving them the opportunity to review and rearrange them according to their own opinion. This provides a structured approach as compared with the traditional rating and ranking. We have used the NAF in different specialties with encouraging results., ${ }^{3,5}$

In conclusion, we support this study to identify and prioritize key areas for simulation-based training. We hope that educators continue to perform structured needs assessment processes before developing important but costly simulationbased courses.

Leizl Joy Nayahangan, RN, MHCM

Copenhagen Academy for

Medical Education and

Simulation, Copenhagen,

Denmark

Lars Konge, MD, PhD, Professor

Copenhagen Academy for Medical Education and Simulation, Copenhagen, Denmark

Faculty of Medicine and the Health Sciences, Copenhagen, Denmark

Keywords: Education, research, simulation

\section{REFERENCES}

1. Kester-Greene N, Hall AK, Walsh CM. Simulation curricular

Correspondence to: Leizl Joy Nayahangan, Copenhagen Academy for Medical Education and Simulation, Copenhagen, Denmark; Email: Leizl.joy. nayahangan@regionh.dk

(C) Canadian Association of Emergency Physicians 
content in postgraduate emergency medicine: A multicenter Delphi study. CFEM 2019; epub, doi:10.1017/cem. 2019.348.

2. Thomas PA, Kern DE, Hughes MT, Chen BY, editor. Curriculum Development for Medical Education: A SixStep Approach. 3 ed. Baltimore: Johns Hopkins University Press; 2015.

3. Nayahangan LJ, Stefanidis D, Kern DE, Konge L. How to identify and prioritize procedures suitable for simulationbased training: Experiences from general needs assessments using a modified Delphi method and a needs assessment formula. Med Teach 2018;40(7):676-83.

4. Nayahangan LJ, Clementsen PF, Paltved C, Lindorff-Larsen KG, Nielsen BU, Konge L. Identifying Technical Procedures in Pulmonary Medicine That Should Be Integrated in a Simulation-Based Curriculum: A National General Needs Assessment. Respiration 2016;91(6):517-22.

5. Nayahangan LJ, Van Herzeele I, Konge L, Koncar I, Cieri E, Mansilha A, et al. Achieving Consensus to Define Curricular Content for Simulation Based Education in Vascular Surgery: A Europe Wide Needs Assessment Initiative. Eur 7 Vasc Endovasc Surg 2019;58(2):284-91. 